\title{
2-D numerical wave Tank by boundary element method using different numerical techniques
}

\author{
Farid Habashi Aliabadi, Parviz Ghadimi*, Seyed Reza Djeddi , Abbas Dashtimanesh \\ Department of Marine Technology, Amirkabir University of Technology \\ *Corresponding author E-mail: pghadimi@aut.ac.ir
}

\begin{abstract}
In this article, numerical modeling of a 2-D wave tank has been investigated by applying completely nonlinear condition for water surface elevation. This has been accomplished based on potential theory, the combined EulerianLagrangian scheme for time marching and using boundary element method. Other physical and numerical attributes of the current work are: physical modeling in time domain, time integration by $4^{\text {th }}$ order Runge-Kutta method, implementation of appropriate condition at the entrance boundary for wave generation, application of artificial dampers at the exit part of the wave tank, and ultimately numerical smoothing of the resulting free surface by using interpolation through spline functions. At the end, effective parameters on the generated wave have been analyzed and the generated wave has also been validated against the result of the linear wave theory.
\end{abstract}

Keywords: Numerical Wave Tank; Boundary Element Method; Wave Damper; Numerical Techniques.

\section{Introduction}

Wave loads are generally the most important forces acting on marine structures including and not limited to ships, platform and wave-breakers. As a result, in order to design the aforementioned structures, loads exerting from waves should be taken into consideration. In the meantime, performing real tests and sea trials would be expensive, risky and in some instances, impossible. This has led research studies toward the design and manufacturing of wave flumes in order to generate desirable waves in laboratories [1, 2]. Nevertheless, wave generation laboratories are relatively expensive and involve further complications. Therefore in the recent years, more attention has been given to the development of numerical methods of wave generation.

In this regard, a multitude of studies have been done for the development of numerical wave tanks (NWT). A numerical wave tank is a generation of numerical simulators which is used for the numerical modeling of nonlinear free-surface waves, hydrodynamics forces and ship motions. In the past two decades, extensive efforts have been made for development of numerical and theoretical techniques. Longuet-Higgins and Cokelet [3] were among the first who suggested the use of numerical wave tank. They used the mixed Eulerian-Lagrangian (MEL) method in which the equations are first solved in the Eulerian framework in order to calculate fluid velocity field. This velocity field is then used for tracking the movements of particles in the Lagrangian framework. Development of the MEL method will lead to the possibility of nonlinear free-surface movements in particular time fractions. Following this, Dommermuth et al. [4] presented a 2-dimensional numerical wave tank for the complex potentials, while Xu and Yue [5] developed a 3D numerical wave tank for the first time and were able to simulate wave overturning phenomenon.

In the framework of these studies, works of Grilli et al. [6] are of high importance. They studied the overturning of waves using their 3-dimensional numerical wave tank which was able to model different seabed geometries. Zhang et al [7] were able to develop a suitable numerical wave tank in order to calculate plunging wave impact on vertical walls. Also in this field, Boo and Kim [8] studied the nonlinear wave diffraction forces acting on the vertical cylinders while Ferrant [9] overviewed the nonlinear forces on cylinders in general using an effective wave and current simulator.

In addition to the works of Longuet-Higgins and Cokelet [3], Vinje and Brevig [10] performed substantial studies in the field of numerical wave tank development. They introduced acceleration field for the first time and showed how the pressure field can be calculated with the least oscillations. Therefore, they were successful in achieving a transient simulation of floating objects in waves. Also in this regard, works of Cointe et al. [11] were among the first in which the acceleration field method was being used. However, the acceleration field method was developed in three different forms. First of all, Tanizawa [12, 13] added the implicit boundary conditions to the acceleration field formulations. 
Later on, Berkvens [14] developed the proposed method to consider three dimensionality effect, and in the past decade, Ikeno [15] and Shirakura et al. [16] implemented the implicit boundary conditions on the 3D numerical wave tank. Following these studies, numerical and theoretical techniques of numerical wave tanks were developed significantly. In the recent years, using numerical wave generators, various offshore and marine problems have been studied and solved. In the analysis of these problems, different numerical details such as implementation of various wave generators, wave dampers, free-surface smoothing strategies among others can be taken into account.

In the present paper, generation and damping of waves using boundary element method (BEM) are studied. Accordingly, a 2-dimensional numerical wave flume is developed using fully nonlinear free-surface boundary conditions. The numerical wave flume is developed using potential theory with Mixed Eulerian-Lagrangian (MEL) method for time marching and boundary element method with constant elements. The Fourth Order Runge-Kutta (RK4) method is used for temporal integrals while 5-points Chebyshev and spline smoothing methods are used for the numerical smoothing of the resulting free-surface.

In addition to this, artificial dampers are used at the exit part of the wave tank in order to avoid wave reflections into the computational domain. The specifications of the wave dampers and their effective length are chosen in a way that will lead to the least reflection of wave and wave energy. Therefore, particular terms are added to the kinematic and dynamic free-surface boundary conditions. Furthermore, the effects of different parameters such as time step, freesurface element length, wave damper efficiency, wave damper effective length and the required number of intervals for the smoothing of the free-surface are studied.

In the sections to come, definition of the problem, the governing equations and the details of the boundary element method are presented. The numerical methods that are used for the accurate simulations are studied and the 2dimensional numerical wave tank is validated. Finally, parametric studies on the effect of different variables on the generated wave are discussed and conclusions are drawn.

\section{Problem setup and mathematical formulations}

Numerical wave tank is consisted for four different surrounding boundaries as depicted in Fig.1. A 2-D wave is generated using a suitable input boundary condition imposed on the left boundary. Also, an artificial damping zone is placed at the right side of the computational domain in order to cancel out the waves approaching the far-right wall. The bottom boundary is set to be the seabed with wall conditions and the free surface boundary is applied at the top of the domain. Assuming the fluid to be incompressible, inviscid and irrotational, the governing equation would be the Laplace equation of the potential function as follows:

$$
\nabla^{2} \phi=0
$$

The Cartesian coordinate system is chosen in a way that the z-axis is stretched vertically from the mean free surface level and the $\mathrm{x}$-axis is stretched downstream-wise.

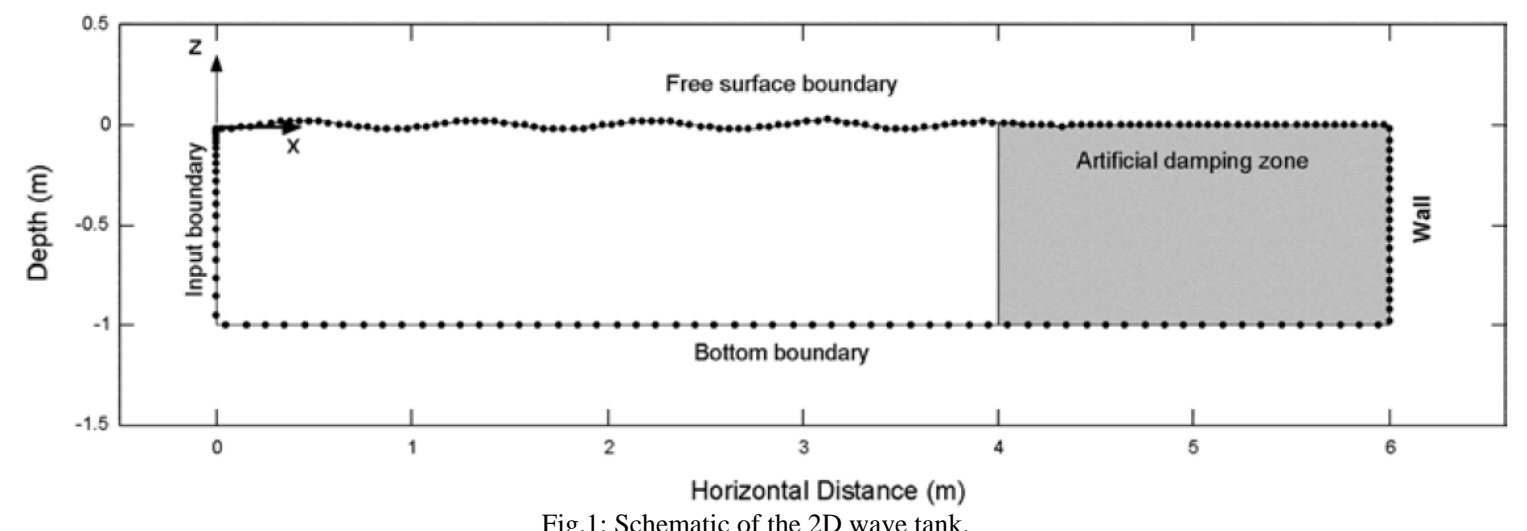

\section{Boundary conditions}

Based on the presented computational domain in the previous section, different boundaries require careful implementation of boundary conditions. In the present section, boundary conditions for the 2-D numerical wave tank are discussed in depth. 


\subsection{Free surface boundary condition}

Both kinematic and dynamic boundary conditions (KFSBC and DFSBC, respectively) should be satisfied at the freesurface boundary. The fully nonlinear dynamic free surface boundary condition is as follows:

$$
\frac{\partial \varphi}{\partial t}=-g \eta-\frac{1}{2}|\nabla \varphi|^{2}-\frac{p_{a}}{\rho}
$$

Also, the fully nonlinear kinematic free surface boundary condition is presented below:

$$
\frac{\partial \boldsymbol{\eta}}{\partial \boldsymbol{t}}=-\nabla \boldsymbol{\phi} . \nabla \boldsymbol{\eta}+\frac{\partial \boldsymbol{\phi}}{\partial \boldsymbol{z}}
$$

\subsection{Inlet boundary condition}

The exact velocity profile of the initial wave is not presented in nonlinear terms. Therefore, the best remedy is to use theoretical wave as the inlet boundary condition. Since the free surface boundary conditions in the computational domain are introduced in terms of nonlinear parameters, the inlet wave would quickly conform to the desired wave shape. Boundary conditions for the linear inlet wave are as follows:

$$
\begin{aligned}
& \frac{\partial \phi}{\partial n}=n_{x} \frac{\partial \phi}{\partial x} \\
& \frac{\partial \phi}{\partial n}=n_{x} \frac{g A k}{\omega} \frac{\cosh [k(z+h)]}{\cosh k \boldsymbol{h}} \cos (k x-\omega t)
\end{aligned}
$$

in which the parameters $\mathrm{A}, \boldsymbol{\omega}, \mathrm{k}$ and $\mathrm{h}$ represent wave amplitude, wave frequency, wave number and water depth, respectively.

\subsection{Wall boundary condition}

In order to satisfy the impermeable characteristic of the wall boundary, the normal gradient of potential function should vanish at this boundary as in equation (5). This boundary condition is imposed on the top and right boundaries of the domain.

$$
\frac{\partial \varphi}{\partial n}=0
$$

\subsection{Artificial damping zone}

At the right side of the wave tank, an artificial damping zone is imposed in order to attenuate the wave energy. In this way, the waves that are approaching the far-right wall boundary will be gradually canceled out. The wave damper types and their effective lengths are chosen in a way that the reflected wave from the damping zone and the wave energy will be minimized. In this regard, required terms are added to both kinematic and dynamic free surface boundary conditions as follows:

$$
\begin{aligned}
& \frac{\delta \phi}{\delta t}=-\boldsymbol{g} \boldsymbol{\eta}-\frac{1}{2}|\nabla \phi|^{2}+\frac{\partial \phi}{\partial z}\left(\frac{\partial \phi}{\partial z}-\frac{\partial \phi}{\partial x} \frac{\partial \eta}{\partial x}\right)-v(x) \phi \\
& \frac{\delta \eta}{\delta t}=\frac{\partial \phi}{\partial z}-\frac{\partial \phi}{\partial x} \frac{\partial \eta}{\partial x}-v(x) \eta
\end{aligned}
$$

in which $v(x)$ is the adjustable attenuation coefficient inside the artificial damping zone. This coefficient is suggested to be a function of the position (x), wave length and wave frequency in most of the research studies. In the present paper, a second-order function of the position ( $\mathrm{x}$ ) is described for the damping coefficient as in

$$
v(x)= \begin{cases}0 & x \leq x_{0} \\ \alpha \omega\left(\frac{x-x_{0}}{\lambda}\right)^{2} & x>x_{0}\end{cases}
$$

in which $\boldsymbol{x}_{0}$ is the longitudinal position at which the damping zone will start to interact while $\alpha$ is the damping factor. It has been found that by setting the damping zone length to one full wave-length and assuming the damping factor to be $\alpha=1$, the wave reflection of the damping zone would be less than $2 \%[17,18]$. 


\section{Boundary element method and boundary value problem (BVP) solution}

The transient velocity potential and the respective free surface profile is calculated using equation (8) based on the discretized form of the integral equation

$\alpha \phi_{i}=\iint_{\Omega}\left(G_{i j} \frac{\partial \phi_{j}}{\partial n}-\phi_{j} \frac{\partial G_{i j}}{\partial n}\right) d s$

in which $\mathrm{G}$ is the Green function that will satisfy the Laplace equation and $\alpha$ is related to the position of the grid nodes. In 2-dimensional problems, the Green function, $G$, is defined as follows [19]:

$\boldsymbol{G}\left(\boldsymbol{x}_{i}, z_{i}, x_{j}, z_{j}\right)=-\frac{1}{2 \pi} \ln R_{1}$

in which $\boldsymbol{R}_{1}$ is the distance between source and each of the boundary nodes.

The integral function is solved using the numerical 4-points Gaussian method and the discretized form of the integral equation is presented as follows [19]:

$\frac{1}{2} \phi_{i}+\sum_{j=1}^{N} \hat{H}_{i j} \phi_{j}=\sum_{j=1}^{N} \boldsymbol{G}_{i j} \frac{\partial \phi_{j}}{\partial n}$

$\boldsymbol{H}_{i j}= \begin{cases}\hat{\boldsymbol{H}}_{i j} & \text { when } i \neq j \\ \hat{H}_{i j}+\frac{1}{2} & \text { when } i=j\end{cases}$

The general form of the above equation is presented below:

$\sum_{j=1}^{N} \boldsymbol{H}_{i j} \boldsymbol{\phi}_{j}=\sum_{j=1}^{N} \boldsymbol{G}_{i j} \frac{\partial \boldsymbol{\phi}_{j}}{\partial \boldsymbol{n}}$

For the problem at hand, after applying the boundary conditions of the potential function in forms of Dirichlet (known $\phi$ ) or Neumann (known $\phi_{n}$ ), the system of equations would be as follows:

$\boldsymbol{H} \boldsymbol{X}=\boldsymbol{G F}$

Matrix $\mathrm{H}$ includes all the known and unknown values of $\phi$ while $\mathrm{G}$ includes all the known and unknown values of $\phi_{n}$. This system of equations would then be ordered in a way that all the known values will be taken to the right hand side of the equation with the unknown values stayed at the left hand side. This system of equations is then solved using the numerical algebraic methods of solution.

$\boldsymbol{A} \boldsymbol{X}=\boldsymbol{B}$

$\boldsymbol{X}=\boldsymbol{A}^{-1} \boldsymbol{B}$

\section{Numerical methods}

In order to solve the transient boundary value problem, different numerical techniques are used. Selected numerical methods and techniques are presented and discussed in this section.

\subsection{Mixed Eulerian-Lagrangian (MEL) method}

The MEL method is used for the simulation of the free surface elevation using the distributed nodes on the boundaries. The implementation of the fully nonlinear solution for the free surface profile using the MEL method was first used by Longuet-Higgins and Cokelet [3]. The time marching method in each time step is described as follows (Fig.2):

1- Laplace equation solution in Eulerian form

2- Modification of the moving boundary nodes and respective values in Lagrangian form 


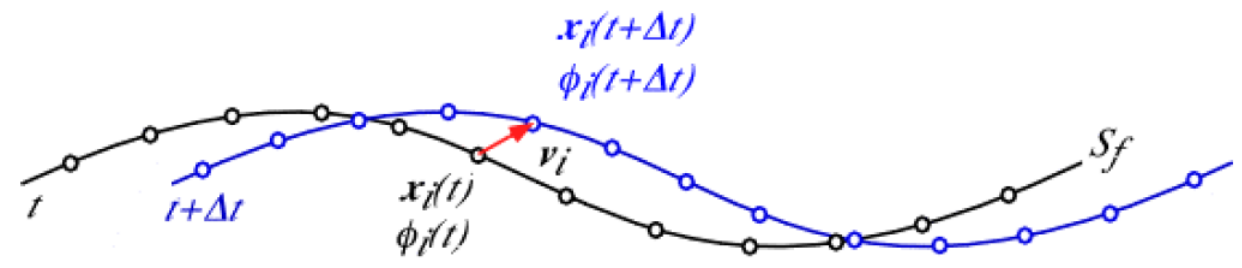

Fig.2: Mixed Eulerian-Lagrangian (MEL) Method.

With the definition of total derivative as in

$(\delta / \delta t)=(\partial / \partial t)+\vec{v} \cdot \nabla$

the nonlinear free surface boundary conditions in Lagrangian form would be as follows:

$\frac{\delta \phi}{\delta t}=-g \eta-\frac{1}{2}|\nabla \phi|^{2}+\nabla \phi \cdot \vec{v}$

$\frac{\delta \eta}{\delta t}=\frac{\partial \phi}{\partial z}-(\nabla \phi-\vec{v}) \cdot \nabla \eta$

Here, $\vec{v}$ and $\eta$ are the nodes velocity and the free surface elevation, respectively.

\subsection{Semi-Lagrangian method}

When the grid nodes represent the movement, the method would be called as semi-lagrangian. In this method, the general approach is to consider the movement of the nodes only in vertical direction and the horizontal movements of the fluid nodes are neglected. Therefore, the velocity vector of the fluid node would be as follows:

$\vec{v}=(0,(\delta \eta / \delta t))$

Consequently, the free surface boundary condition is presented in equation (17).

$$
\begin{aligned}
& \frac{\delta \phi}{\delta t}=-\boldsymbol{g} \eta-\frac{1}{2}|\nabla \phi|^{2}+\frac{\partial \phi}{\partial z}\left(\frac{\partial \phi}{\partial z}-\frac{\partial \phi}{\partial x} \frac{\partial \eta}{\partial x}\right) \\
& \frac{\delta \eta}{\delta t}=\frac{\partial \phi}{\partial z}-\frac{\partial \phi}{\partial x} \frac{\partial \eta}{\partial x}
\end{aligned}
$$

The semi-lagrangian method would complicate the free surface boundary conditions, while on the other hand, the computational grid would not require any modification or refinement process. In the developed numerical wave tank that is presented in the current study, both methods of MEL and semi-Lagrangian are used.

\subsection{Computational grid modification process}

When the new free surface is set in each time step, nodes can get so close to or so far from each other which will lead into numerical instabilities. Therefore, for avoiding this instability, the free surface and the computational grid should be reconstructed in a modification process. For the interpolation of the new free surface nodes, the square strips approximation method is used. The reconstruction or modification of the computational grid is performed in three stages. In the first stage, the spline approximation of the free surface profile is calculated using the element linear length as a parameter of interest. In the second stage, the arc length of the free surface profile is determined and the spline approximation is formulated using the calculated arc length. Finally, the nodes on the free surface profile would be relocated based on the representation of the boundary using elements with equal length. However, the element lengths can be considered gradually increasing using a growth rate [20].

\subsection{Ramp function}

The ramp function is used for wave modeling on the inlet boundary. This function will prevent the abrupt behavior of the wave maker which will result in more stability of the generated waves inside the computational domain. Therefore, using the ramp function at the inlet boundary will lead to a more stable solution. This function is imposed on a time range that is a factor of the wave period. In the present study, this time range is set to be 2 wave periods. 
$r(t)= \begin{cases}1 & \text { for } t>2 T \\ \left\{1-\cos \left(\pi \frac{t}{2 T}\right)\right\} / 2 & , \text { for } t \leq 2 T\end{cases}$

\subsection{Time step restriction}

In numerical simulations, distance between the intermediate nodes (i.e. $\Delta \boldsymbol{x}$ ) and the time step ( $\Delta t$ ) should be chosen in such a way to avoid numerical instabilities. Dommermuth and Yue [21] studied the Von-Neumann stability analysis for the application of the fourth order Runge-Kutta (RK4) method to linearize free surface conditions and presented the courant criterion as follows:

$\Delta t^{2} \leq \frac{8}{\pi} \frac{\Delta x}{g}$

As stated by Kim et al. [22], equation (19) is expected to be also valid for nonlinear free surface conditions. This equation gives the maximum allowable time step size $(\Delta t)$ based on the element size $(\Delta x)$ but does not necessarily restrict this value.

\subsection{Time marching method}

In the framework of the Mixed Eulerian-Lagrangian (MEL) method, velocity potentials on the free surface and the normal derivative of the potential field on the boundaries are known. The velocity potential and the free surface elevation will be calculated using the temporal integration of the free surface boundary conditions based on the time step size $(\Delta t)$. This process is known as the time marching procedure. In this regard and in the present study, the fourth order Runge-Kutta (RK4) method, as one of the most stable methods for nonlinear conditions in simulation, is used.

\subsection{Free surface smoothing}

The free surface smoothing procedure is performed at each time step. The numerical instabilities can be overcome using this technique. In the time marching process, sawtooth instabilities appeare on the free surface profile. One reason for this behavior is due to the nature of the numerical integrals which were solved using numerical methods. Another reason would be the physical nature of the problem at hand.

Sawtooth instabilities can be avoided using smoothing techniques. In the present numerical wave tank, two methods of 5-points chebyshev [24] and 5-points third-order spline [25] methods are used for the temporal integration on the free surface.

\section{Effect of different parameters on the simulations of numerical wave tank}

As discussed in the previous sections, different numerical methods and techniques are required for accurate simulation of this problem. Implementing each of these methods would cause particular difficulties for the generation of waves. Therefore, the stability of the temporal integrals and the accuracy of the obtained results would be largely affected by the time step size $(\Delta t)$ and some other leading factors. In general, the accuracy of the generated wave and its compatibility with theoretical results would require a suitable combination of the following parameters:

1- Time Step Size

2- Free Surface Element Size

3- Wave Damping Factor

4- Wave Damper Effective Length

5- Number of Smoothing Intervals

In the present study, in order to validate the developed 2-dimensional numerical wave tank, the effects of the above mentioned parameters are studied in details. Finally a suitable value for each of these parameters can be determined. In these simulations, a computational domain of 6 meters long is used and the wave specifications are as follows:

a) the water depth and the wave length are both 1 meter,

b) wave amplitude is $0.02 \mathrm{~m}$ and the wave period is set to be 0.8005 seconds.

c) the simulations are performed for 18 seconds and

d) based on the wave length and the water depth in the framework of the wave theory, the wave tank can be considered as deep water: 
$\frac{d}{L}=\frac{1}{1}=1>\frac{1}{2}$

\subsection{Effect of time step}

Using different time step sizes while maintaining the number of free surface nodes at a constant value will result in contrasting behaviors of the generated wave as shown on Fig.3. The simulations are done for two different time step sizes with the total duration of 18 seconds. It is shown that the time step will affect the computational results and the solver is highly dependent on the time step size.

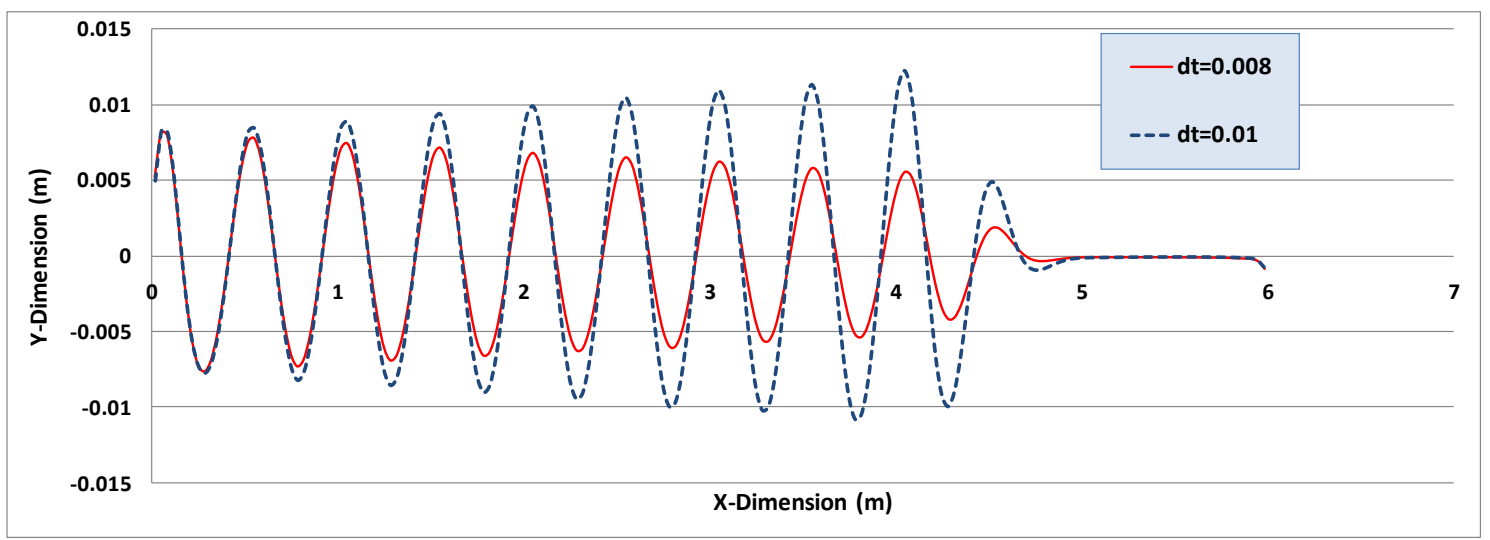

Fig.3. The effect of the time step size on the generated wave profile

\subsection{Effect of free surface element size}

In order to study the effects of a fine free surface grid sizing, the number of free surface elements has been varied while keeping the time step size as a constant value. The results for two different element sizes are shown on Fig.4. In this figure, $\boldsymbol{n}_{1}$ is the number of free surface nodes. It is clear that the numerical solution is largely affected by the number of these elements and therefore extra care should be given to the determination of this value.

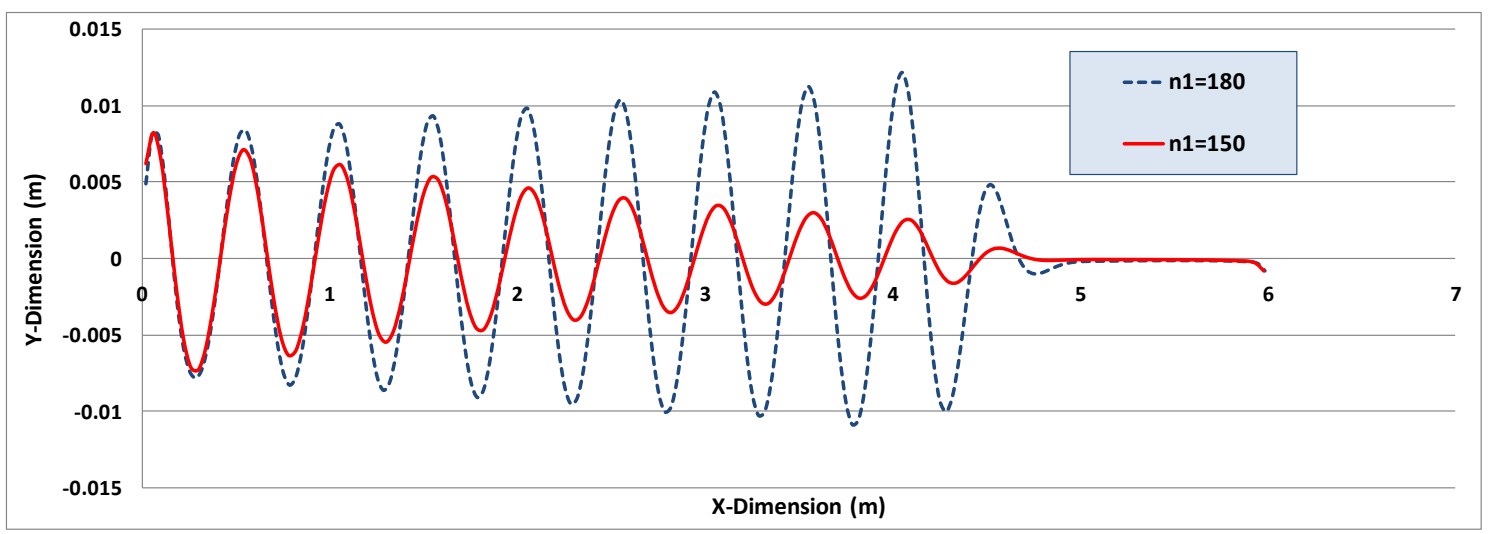

Fig.4: The effect of the free surface element size on the generated wave profile

\subsection{Effect of wave damping factor}

As stated in section 3 and explained in details in subsection 3.4, wave damper or the artificial numerical damping zone has two main parameters. Damping factor $\alpha$ is one of these parameters which is shown to be largely affecting the numerical solution, as shown in Fig.5. 


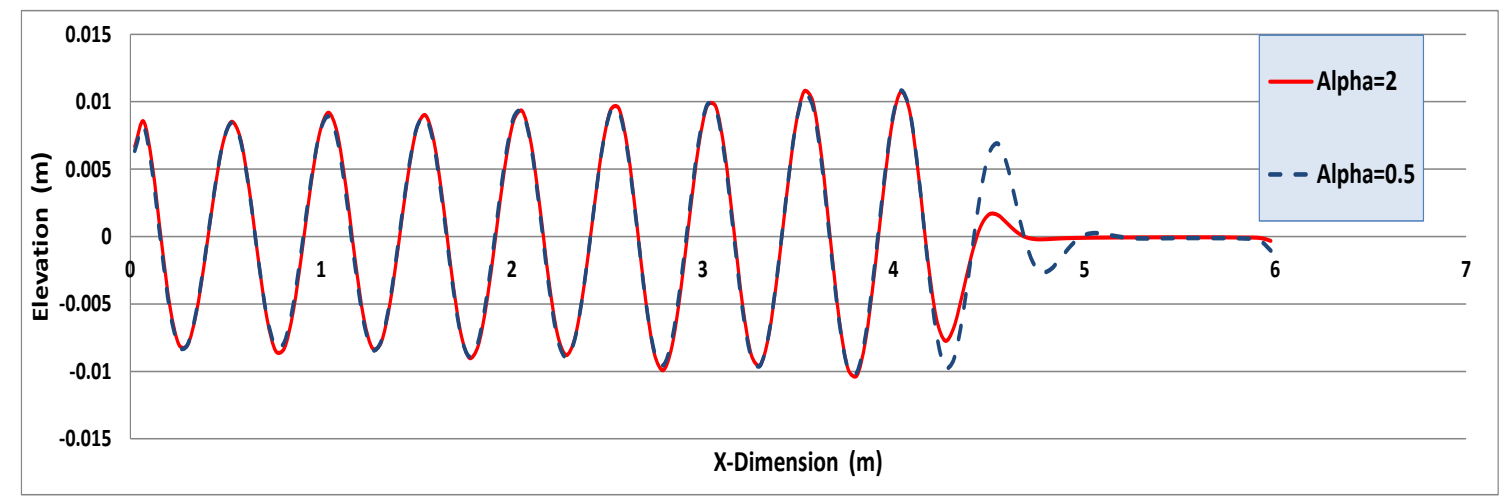

Fig.5: The effect of the wave damping factor on the generated wave profile

\subsection{Effect of wave damper effective length}

Another parameter which plays a significant role in the damping behavior of the artificial number damping zone is the damper effective length. It is shown in Fig.6 that this parameter will affect the obtained numerical solution and a longer damping zone will be able to cancel out the approaching waves, more effectively.

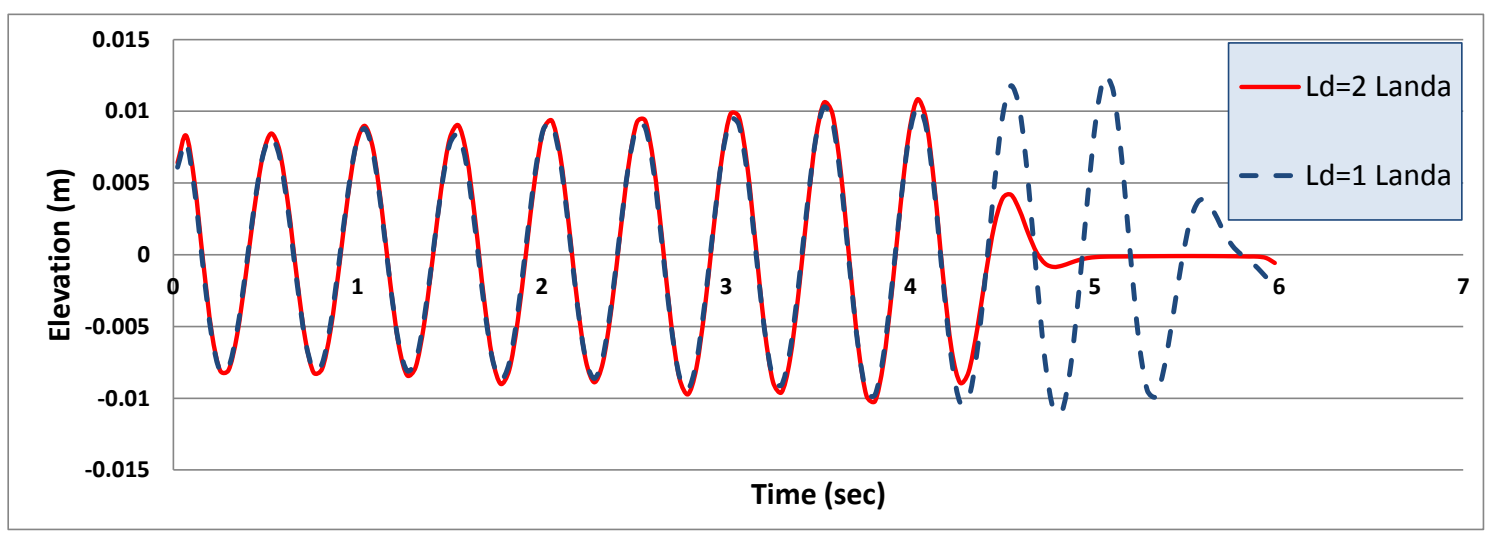

Fig.6: The effect of the wave damper effective length on the generated wave profile

\subsection{Effect of number of smoothing intervals}

Number of the smoothing intervals is a parameter that shows the intervals in which the smoothing process will happen. In order to study the effects of this parameter on the numerical solution, two different intervals are chosen. First, the smoothing process is performed at each time step and in another simulation this process is performed in every two time steps. The results which are depicted in Fig.7 show that with quicker smoothing, wave will not be generated effectively and with small number of smoothing intervals, the numerical solver will be unstable.

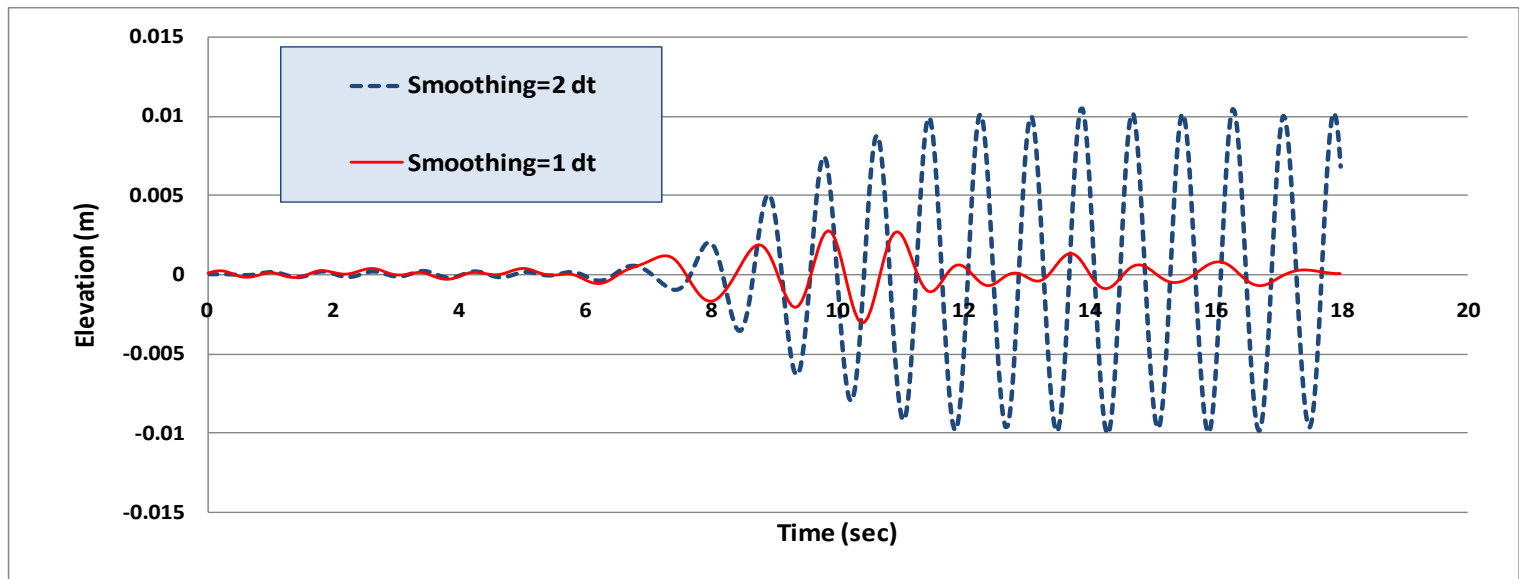

Fig.7: The effect of the number of the smoothing intervals on the generated wave profile 


\subsection{Effect of ramp function time range}

As presented in the previous sections, a ramp function is used for the wave generator boundary condition which will prevent the abrupt motions of the generator. The time range for imposing this function is changed in order to study its effect on the stability and consistency of the numerical solver. In Fig.8, the time range $\boldsymbol{T}_{\boldsymbol{m}}$ shows how long the ramp function will be gradually imposed on the boundary until it reaches a constant value. Decrease in the time range $\boldsymbol{T}_{\boldsymbol{m}}$ will change the ramp function behavior until the time $\boldsymbol{T}_{\boldsymbol{m}}=0$ at which, it will act like a step function. It is shown that an abrupt motion of the wave maker can lead to an unstable generated wave profile and with larger time ranges, $\boldsymbol{T}_{\boldsymbol{m}}$, wave will be generated later but with less inconsistency and more stability.

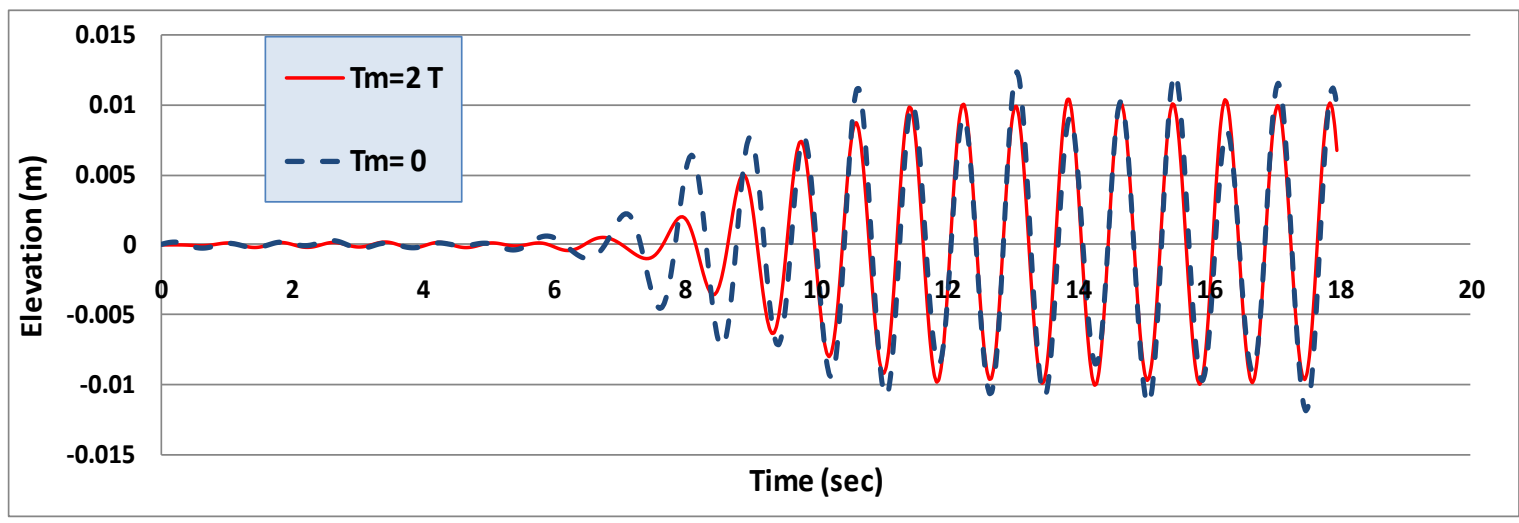

Fig.8: The effect of the ramp function time range on the generated wave profile

Based on an extensive parametric study that was performed on these parameters that would largely affect the numerical solution, a suitable combination and ideal determination of these values have been obtained. Based on the wave tank and the wave profile specifications, the following parameters are suggested:

1- Time step size: $\Delta t=0.0175$ (sec)

2- Number of free surface nodes: $\boldsymbol{n}_{1}=130$

3 - Number of nodes on the end wall of the wave tank: $\boldsymbol{n}_{2}=30$

4- Number of nodes on the wave maker or the inlet boundary: $\boldsymbol{n}_{3}=50$

5- Number of nodes on the wave tank bottom: $\boldsymbol{n}_{4}=100$

6- Number of smoothing intervals: every two time steps $($ Smoothing $=2 \Delta t)$

7- Time range for the ramp function: $\boldsymbol{T}_{\boldsymbol{m}}=2 \boldsymbol{T}$

8- Artificial Damping Zone: $\boldsymbol{L}_{\boldsymbol{d}}=2 \lambda, \boldsymbol{\alpha}=1.0$

In the following section, validation of the $2 \mathrm{D}$ generated wave is performed against the theoretical results.

\section{Validation of the numerical wave tank}

In this section, the generated regular wave inside the numerical wave tank is validated using the theoretical wave profile. As shown in Fig.9, the generated wave inside the tank appears after about 8 seconds and at around $\boldsymbol{t}=12.0$ (sec) Numerical and analytical results are in good agreement which verifies the numerical stability and accuracy of the developed BEM solver. Results are probed at a location of $\boldsymbol{x}=3.0(\boldsymbol{m})$ from the origin. It is worth mentioning that, with the present specifications of the wave profile and the tank, the generated wave will meet the physical conditions after 12 seconds. The obtained results prove the accuracy of the numerical solver in generating regular waves inside the tank. Also, the propagation of the generated wave inside the tank at two different time durations are shown in Fig.10 which also vouch for the efficiency of the present solver in maintaining its accuracy over the simulations. 


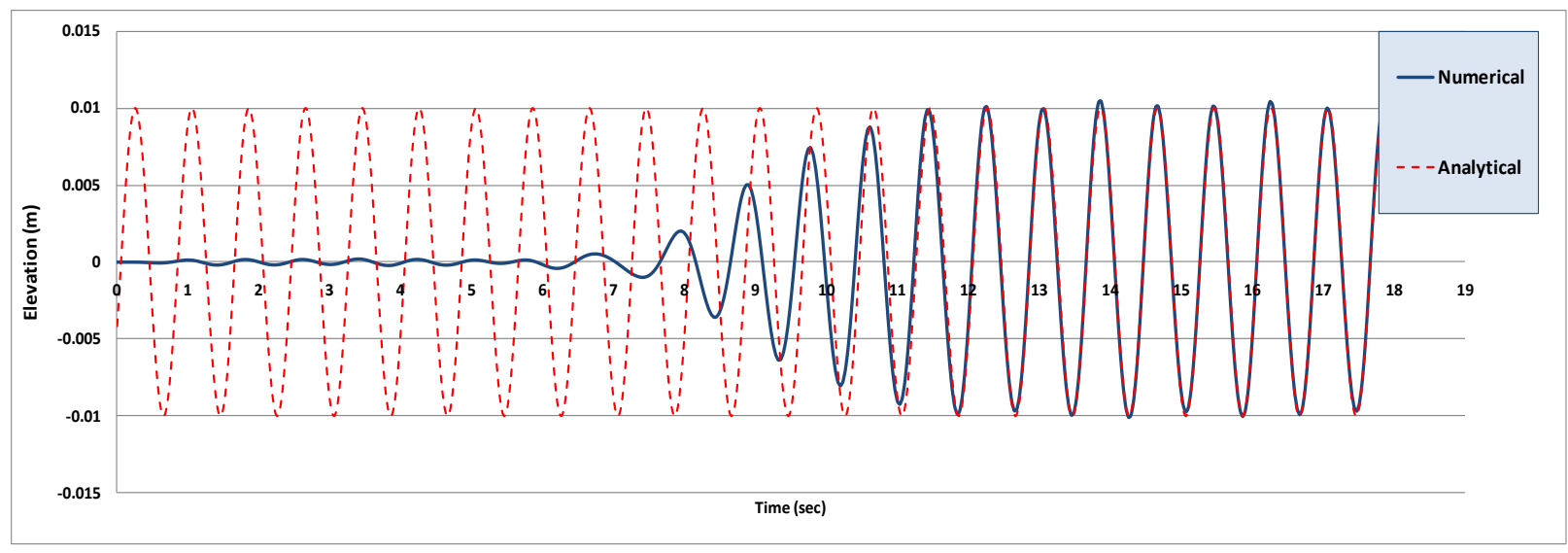

Fig.9. Comparison of the generated numerical wave with the theoretical profile for the probing location at $\boldsymbol{x}=3.0(\boldsymbol{m})$.

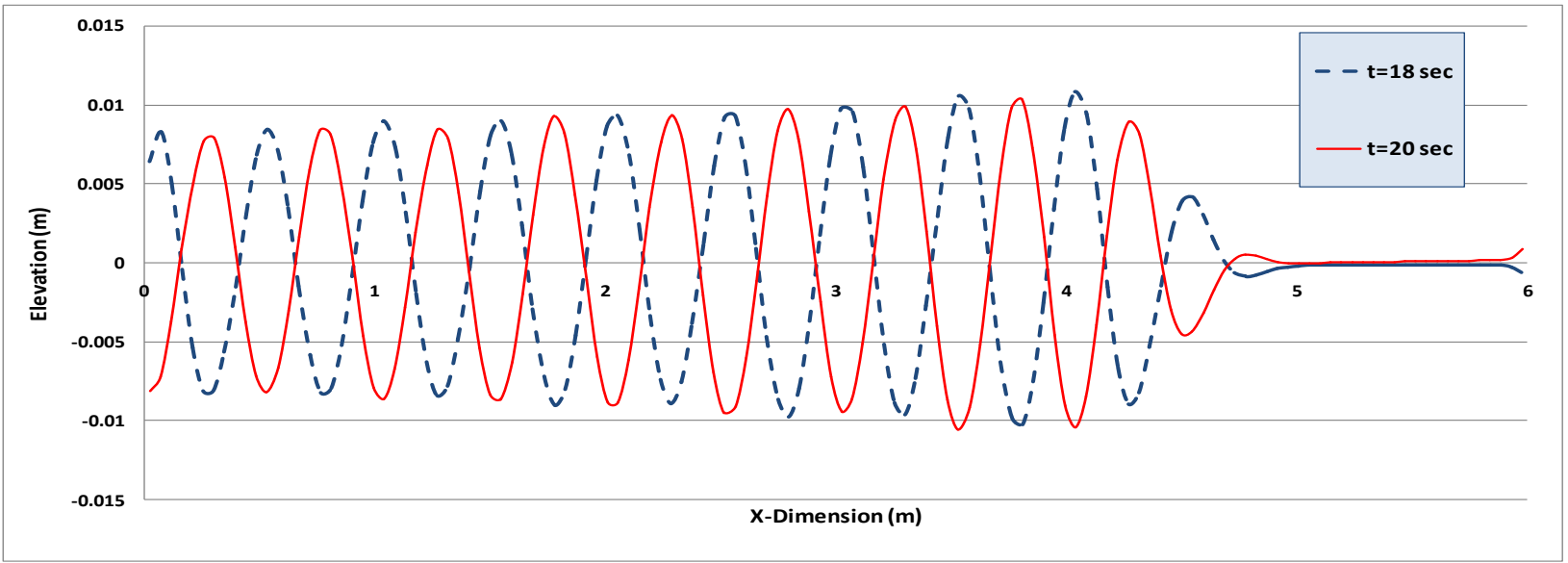

Fig.10: Propagation of the generated wave inside the numerical wave tank over time

\section{Conclusion}

In the present study, a 2-dimensional numerical wave tank is developed. With the assumption of an inviscid, irrotational and incompressible fluid, the Laplace equation of the potential function is the governing equation of the solver. Using a suitable inlet boundary condition as the wave maker of the tank and with nonlinear free surface boundary conditions, the generation of waves is studied. Also, an artificial damping zone is placed at the exit part of the tank in order to cancel out the generated waves which approach the far-right wall boundary of the computational domain. This damping zone will prevent the generated wave from being reflected back into the domain. The boundary element method (BEM) with constant sized elements is used for numerical solution of the governing equation. Additionally, the fourth order Runge-Kutta (RK4) method is used for the transient solution of the equations and for performing temporal integrations. The effects of different parameters on the consistency and accuracy of the developed solver are studied. In this regard, with an initial node seeding with a constant element size, increase of the time step causes the wave amplitude to increase and the amplitude will get even higher as the generated wave propagate toward the end of the tank. Also, same results can be achieved by increasing the number of free surface nodes or decreasing the element size. It has been observed that the obtained numerical results are largely affected by the number of free surface nodes and it can also be altered with changes in number of nodes on the inlet boundary (wave maker) while the number of nodes on the bottom and exit boundaries are not important for the accuracy of the numerical solutions. In the framework of an extensive study on the effects of the different parameters, the number of smoothing intervals plays a significant role on the efficiency and accuracy of the obtained results. It is shown that when the smoothing process is performed at longer intervals, wave would not be generated and when it happens more quickly, the solution would get unstable.

Finally, the developed numerical wave tank is validated against the theoretical results. Comparison shows that the BEM solver which is developed and presented in the current study is in good agreement with the theoretical wave profiles and therefore the numerical wave tank exhibits good consistency and accuracy for the generation and propagation of 2 dimensional regular waves. 


\section{References}

[1] Beck, R.F., Cao, Y. and Lee, T.H., (1993), "Fully nonlinear water wave computations using the desingularized method", Proc. of 6th Int. Conf. on Ship Hydro., pp.3-20.

[2] Grilli,S. and Horrillo,J., (1997), "Numerical generation and absorption of fully nonlinear periodic waves", J. Eng. Mech., ASCE, Vol.123, pp.1060-1069.

[3] Longuet-Higgins, M, \& Cokelet, E. D, (1976). "The deformation of steep surface waves on water". I. A numerical method of computation. Proc. R. Soc. Lond. A 350, 1-26.

[4] Dommermuth, D.G., Yue, D.K.P., Lin, W.M., Rapp, R.J., Chan, E.S. and Melville, W.K., (1988), "Deep water plunging breakers: a comparison between potential theory and experiments", J. Fluid Mech., Vol.189, pp.423-442.

[5] Xu, H. and Yue, D.K.P., (1992), "Numerical study of three dimensional overturning waves", Proc. 7th Workshop on Water Waves and Floating Bodies, pp.303-307.

[6] Grilli, S., Guyenne, P. and Dias, F. (2000), "Modeling of overturning waves over arbitrary bottom in 3D numerical wave tank", Proc. 10th ISOPE Conf., Vol.3, pp.221-228

[7] Zhang, S., Yue, D.K.P. and Tanizawa, K., (1996), "Simulation of plunging wave impact on a vertical wall”, J. Fluid Mech., Vol.327, pp.221254

[8] Boo, S.Y. and Kim, C.H., (1996), "Fully nonlinear diffraction due to a vertical circular cylinder in a 3- D HOBEM Numerical Wave Tank", Proc. 6th ISOPE Conf., Los Angeles, USA, Vol.3, pp.76-84

[9] Ferrant, P., (1998), "Run-up on a cylinder due to waves and current: Potential flow solution with fully nonlinear boundary conditions", Proc. 8th ISOPE Conf., Vol.3, pp.332-339

[10] Vinje, T., and Brevig, P., (1981b), "Nonlinear, two-dimensional ship motions", Norwegian Hydrodynamic Laboratories, Report R112.81,Trondheim, Norway., pp.1-97

[11] Cointe, R., Geyer, P., King, B., Molin, B. and Tramoni, M. (1990), "Nonlinear and linear motions of a rectangular barge in perfect fluid", Proc. of the 18th Symp. On Naval Hydro., AnnArbor, Michigan, pp.85-98.

[12] Tanizawa, K., (1995a), "A Nonlinear Simulation Method of 3-D body Motions in Waves (1st Report)", J. Soc. Nav. Arch. Japan, vol.178, pp.179-191.

[13] Tanizawa, K., (1996a), "Nonlinear simulation of floating body motions in waves", Proc. of 6th ISOPE conference, Los Angeles, California, Vol.3, pp.414-420.

[14] Berkvens, P. J. F. (1998), "Floating bodies interacting with water waves", Ph.D. thesis, University of Twente, The Netherlands, pp.1-161.

[15] Ikeno,M., (2000), "A numerical model for 3-D floating body motion in nonlinear waves usingthe BEM", Proc. 10th ISOPE Conf., Vol.3, pp.201-213.

[16] Shirakura, Tanizawa, Naito (2000), "Development of 3-D fully nonlinear numerical wave tank to simulate floatingb odies interacting with water waves", Proc. 10th ISOPE Conf., Seattle, Vol.3, pp.253-262.

[17] Cl'ement,A.H., (1999b), "Benchmark test cases for numerical wave absorption”, Proc. 9th ISOPE Conf., Vol.3, pp.266-289.

[18] Contento G, Codiglia R, D’Este F, (2001). "Nonlinear effects in 2D transient nonbreaking waves in a closed flume ". Applied Ocean Research, 23:3-13

[19] S. S. Bayin (2006), Mathematical Methods in Science and Engineering, Wiley, Chapters 18 and 19.

[20] Sun, Hui, (2007), "A Boundary Element Method Applied to Strongly Nonlinear Wave-Body Interaction Problems" PhD Thesis, Norwegian University.

[21] Dommermuth, DG, Yue, DKP, (1987). "Numerical simulation of nonlinear axisymmetric flows with a free surface", J. Fluid Mech. 178: 195219

[22] Kim, CH, Clement, A.H, Tanizawa, K, (1999). "Recent research and development of numerical wave tanks" A Review. Int. J. Offshore and Polar Eng., ISOPE, 9: 241-256

[23] Tanizawa, Katsuji, (2000), "The State of The Art on Numerical Wave Tank", 4th Osaka Colloquium on Seakeeping Performance of Ships, PP 95-114.

[24] Koo, Weoncheol, (2003), "Fully Nonlinear Wave-Body Interaction by a 2D Potential Numerical Wave Tank" PhD Thesis, Texas A\&M University.

[25] Maruo, H, Song, W, (1994). "Nonlinear analysis of bow wave breaking and deck wetness of a highspeed ship by the parabolic approximation". In: Proc. 20th Symposium on Naval 